\title{
PEA3/ETV4-related transcription factors coupled with active ERK signalling are associated with poor prognosis in gastric adenocarcinoma
}

\author{
R Keld ',2, B Guo', P Downey ${ }^{3}$, R Cummins ${ }^{4}$, C Gulmann ${ }^{3}$, YS Ang ${ }^{2}$ and AD Sharrocks*,I \\ 'Faculty of Life Sciences, University of Manchester, Michael Smith Building, Oxford Road, Manchester MI 3 9PT, UK; ${ }^{2}$ Faculty of Medicine, University of \\ Manchester, Oxford Road, Manchester MI 3 9PT, UK; ${ }^{3}$ Department of Histopathology, Beaumont Hospital, Dublin, Ireland; ${ }^{4}$ Department of Pathology, \\ Royal College of Surgeons in Ireland, Dublin, Ireland
}

BACKGROUND: Transcription factors often play important roles in tumourigenesis. Members of the PEA3 subfamily of ETS-domain transcription factors fulfil such a role and have been associated with tumour metastasis in several different cancers. Moreover, the activity of the PEA3 subfamily transcription factors is potentiated by Ras-ERK pathway signalling, which is itself often deregulated in tumour cells.

METHODS: Immunohistochemical patterns of PEA3 expression and active ERK signalling were analysed and mRNA expression levels of PEA3, ER8I, MMP-I and MMP-7 were determined in gastric adenocarcinoma samples.

RESULTS: Here, we have studied the expression of the PEA3 subfamily members PEA3/ETV4 and ER8I/ETVI in gastric adenocarcinomas. PEA3 is upregulated at the protein level in gastric adenocarcinomas and both PEA3/ETV4 and ER8//ETVI are upregulated at the mRNA level in gastric adenocarcinoma tissues. This increased expression correlates with the expression of a target gene associated with metastasis, MMP-I. Enhanced ERK signalling is also more prevalent in late-stage gastric adenocarcinomas, and the co-association of ERK signalling and PEA3 expression also occurs in late-stage gastric adenocarcinomas. Furthermore, the coassociation of ERK signalling and PEA3 expression correlates with decreased survival rates.

CONCLUSIONS: This study shows that members of the PEA3 subfamily of transcription factors are upregulated in gastric adenocarcinomas and that the simultaneous upregulation of PEA3 expression and ERK pathway signalling is indicative of late-stage disease and a poor survival prognosis.

British Journal of Cancer (201 I) I 05, 124-130. doi:I0.1038/bjc.201 I.I87 www.bjcancer.com

Published online 14 June 201 I

(c) 201। Cancer Research UK

Keywords: PEA3; ER8I; ETVI; ETV4; MMPI; gastric cancer

Gastric cancer is the second commonest cause of a cancer death worldwide. In Western populations, the incidence has been steadily declining over the past decades (Samalin and Ychou, 2007; Bosetti et al, 2008). This is thought to be a result of a decreasing incidence of Helicobacter pylori by chlorination of drinking water and policies for antibiotic eradication. Despite this reduction, in England gastric cancer remains ranked as the sixth commonest cause of a cancer death (Rachet et al, 2009). This is largely attributable due to the late presentation of the disease and this limits treatment options. Five-year survival is excellent if diagnosed at an early stage and population screening by upper gastrointestinal endoscopy has increased the rates of early detection and subsequent prognosis in Japanese populations (Nosho et al, 2005; Tan and Fielding, 2006). In Western populations, the incidence of gastric cancer is much lower and population screening is not economically viable. As a result, gastric adenocarcinomas are usually diagnosed at an advanced

*Correspondence: Professor AD Sharrocks;

E-mail: a.d.sharrocks@manchester.ac.uk

Received I 8 January 201 I; revised 27 April 201 I; accepted 5 May 20I I; published online 14 June 201 I stage and typical 5-year survival is $15 \%$. Cytotoxic chemotherapy regimens are largely ineffective in halting the disease (Findlay et al, 1994; Mackay et al, 2001; Tebbutt et al, 2002). Even when surgery is possible, neoadjuvant cytotoxic chemotherapy improves 5-year survival rates modestly from 20 to $36 \%$ (Cunningham et al, 2006). New treatments and targets for drug therapies are needed to improve outcomes further.

PEA3/ETV4 is a member of the PEA3 subfamily of ETS-domain transcription factors. This subfamily also includes ER81/ETV1 and ERM/ETV5. The ETS domain determines DNA-binding specificity, it is highly conserved in all three PEA3 family proteins and this suggests that they regulate similar promoters (de Launoit et al, 1997). This has been shown to be the case with other ETS family members, where both promoter-specific and redundant binding modes are operative (Hollenhorst et al, 2007; Boros et al, 2009). The PEA3 family is important in development, with particular recent emphasis on the nervous system (de Launoit et al, 1997; Oikawa and Yamada, 2003; Sharrocks, 2001, Vrieseling and Arber, 2006). However, much interest has surrounded PEA3 proteins because of their association with cancer. The proteins are generally expressed at low levels in normal adult tissues, however, in cancer tissues, mRNA and protein expression is often much 
higher. PEA3 is associated with a variety of cancers including colon, breast, ovarian, prostate and oesophageal cancer (de Launoit et al, 2000; Horiuchi et al, 2003; Cowden Dahl et al, 2007; Tomlins et al, 2007; Keld et al, 2010). ER81 has been associated with prostate, oesophageal and gastrointestinal stromal tumours (Cai et al, 2007; Chi et al, 2010; Keld et al, 2010). Where present, tumours are generally more aggressive, advanced and prognosis is worse. It has been established that PEA3 proteins are normally under auto-inhibitory control (reviewed in Sharrocks, 2001). For optimal transcriptional activity, PEA3 proteins require activation by post-translational modification from mitogenic signalling pathways. ERK MAP kinase signalling is important for PEA3 and ER81 activation through direct phosphorylation and subsequent SUMOylation (reviewed in de Launoit et al, 2006; Guo et al, 2007; Brown et al, 1998; Janknecht, 2003; Goel and Janknecht, 2004; Guo and Sharrocks, 2009). Various co-factors such as $\beta$ catenin, c-Jun, p300 and LPP enhance the transcriptional activity of PEA3 in cell line models (Gum et al, 1996; Crawford et al, 2001; Janknecht, 2003; Liu et al, 2004; Guo et al, 2006; Matsui et al, 2006). This indicates that additional factors are needed for optimal activity. The poor prognosis associated with tumours that express PEA3 proteins is thought to be due to the activation of genes encoding matrix metalloproteases (MMP-1, 2, 7, 9, 11, 13 and 14) (Habelhah et al, 1999; Horiuchi et al, 2003; Cai et al, 2007; Cowden Dahl et al, 2007), cell cycle regulators (Cyclin D3; Jiang et al, 2007) and the production of growth factors receptors (HER-2; O'Hagan and Hassell, 1998) and mediators of angiogenesis (COX2 and VEGF; Howe et al, 2001; Hua et al, 2009; Liu et al, 2004).

In gastric adenocarcinoma cell line models, PEA3 increases $M M P-1$ and MMP-7 expression and stimulates invasion in vitro (Yamamoto et al, 2004). The ERK MAPK kinase pathway has been shown to activate PEA3 and increase $M M P-1$ levels by incubating gastric cancer cells with $H$. pylori (Wu et al, 2006). In a recent study, HER-2 was shown to drive $M M P-1$ expression and gastric cancer cell invasion (Bao et al, 2010), but the role of PEA3 in this process was not investigated. Our understanding of the PEA3 family and ERK MAPK signalling in gastric adenocarcinoma is limited. One previous study looked at PEA3 in this context and demonstrated that PEA 3 mRNA expression is associated with poor prognosis and disease recurrence in gastric adenocarcinoma in a Japanese population (Yamamoto et al, 2004). The other subfamily members ER81 and ERM did not influence disease stage or outcome. The role of MAPK signalling was not investigated in this study. No previous studies have investigated PEA3 or ER81 in a Western population of gastric adenocarcinomas. Only one study has investigated the status of ERK MAP kinase signalling in gastric adenocarcinomas. Here, a low occurrence was found but clinical correlations were not made (Feng et al, 2008). A three-way association to generate an ERK-PEA3-MMP axis has been described in cell lines derived from ovarian, oesophageal and gastric cancer (Wu et al, 2006; Cowden Dahl et al, 2007; Keld et al, 2010). Moreover, both PEA3 and MMP-1 were shown to be upregulated in patient-derived early-stage gastric cancer samples from a Japanese population ( $\mathrm{Wu}$ et al, 2006). This upregulation was potentiated in the presence of $H$. pylori infection, thereby suggesting a role for $H$. pylori-induced ERK pathway activity in driving their expression. Here, we revisited this issue and examined more directly whether the ERK-PEA3-MMP axis is operative in gastric adenocarcinoma tissue.

Primarily, we investigated the expression of the PEA3 subfamily members PEA3 and ER81 in gastric adenocarcinomas. Second, we investigated upstream pathways by measuring ERK MAP kinase activity and downstream pathways by determining the levels of the putative targets $M M P-1$ and $M M P-7$. We found that increased expression of both PEA3 and ER81 are associated with gastric adenocarcinomas. Furthermore, PEA3 and ER81 expression are associated with elevated $M M P-1$ and $M M P-7$ expression. In isolation, PEA3 expression was not associated with poor prognosis or an advanced disease stage. In adenocarcinoma tissue, PEA3 expression in combination with elevated ERK MAP kinase signalling is associated with an advanced disease stage and a reduced survival compared with specimens with ERK MAP kinase signalling without PEA3 or in specimens with PEA3 expression in isolation. This study indicates that the knowledge of transcription factor expression in combination with the activity of the upstream signalling pathways may also be important for patient selection to tailor therapy more effectively to improve treatment response.

\section{MATERIALS AND METHODS}

\section{Tissue collection}

Ethical approval was granted by Wrightington Wigan and Leigh Ethics Committee, UK in 2004. Tissue was collected from 40 patients with gastric adenocarcinomas and 15 healthy controls, and clinical characteristics recorded as described previously (Keld et al, 2010).

\section{RNA isolation and RT - PCR analysis}

RNA was extracted, its' integrity determined and subsequent realtime RT - PCR performed for PEA3, ER81, MMP-1, MMP-7 and $18 S$ RNA as described previously (Keld et al, 2010). Data are presented relative to $18 \mathrm{~S}$ RNA levels in the same samples. For relative comparison of mRNA levels from tissue specimens, data were further normalised to the level of each gene in a stock standard concentration of RNA isolated from OE33 (for MMP-1 and MMP7), SW480 (for PEA3) and Flo1 (for ER81) cells. This latter normalisation enabled experiments performed at different times to be compared as mRNA concentrations were calculated relative to the same standard stock mRNA preparation. The cell lines were cultured and lysed as described previously (Keld et al, 2010).

\section{Immunohistochemistry}

Tissue microarray blocks were constructed from surgical resection tumour blocks and biopsies as described previously (Keld et al, 2010). Three arrays were constructed for each case and stained with PEA3 (Santa Cruz Biotechnology, Santa Cruz, CA, USA; Sc 113) and phosphorylated ERK (P-ERK) antibodies (New England Biolabs, Ipswich, MA, USA; \#437S) at a $1: 20$ and $1: 100$ dilution, respectively. A negative control slide was tested without the primary antibody to detect any background staining or falsepositive results. Three cores for each specimen were constructed and scored by two expert histopathologists blinded to the clinical details. A positive score was determined by the presence of positive staining in 5\% of tumour cells. An intensity score of 1-4 was also determined. Moderate to high expression (intensity score 3 and 4) was judged to be present if staining was visible easily at $\times 20$ magnification. The highest score in the triplet of cores was recorded. We took moderate to high expression as positive for PEA3 protein expression and P-ERK occurrence.

\section{Statistical analysis}

The software package SPSS 15.0 was utilised to analyse data. The $t$-test was used to compare means of continuous variables. Fisher's exact test was used to compare discrete variables. Kaplan-Meier analysis using the Breslow Wilcoxan test was used to calculate survival curves by univariate analysis. Significance was accepted to be present with a $P$-value $<0.05$. 


\section{RESULTS}

PEA3 and ER81 are associated with gastric adenocarcinomas

We recently demonstrated that PEA3 family members are associated with oesophageal adenocarcinomas (Keld et al, 2010) and wanted to know whether they are also associated with gastric adenocarcinoma. First, we performed immunohistochemistry to assess the expression of PEA3 at the protein level using a TMA constructed from 39 gastric adenocarcinoma and 15 non-cancer tissue specimens. Samples were then scored according to PEA3 levels and were considered as positive if they had moderate-high PEA3 protein levels (Figure 1A; bottom panels). Moderate to high PEA3 protein expression was present in $49 \%$ of adenocarcinomas compared with $10 \%$ in non-cancer tissue $(P$-value $=0.018)$ (Figure 1B). However, we did not identify a relationship between PEA3-expressing tumours with $H$. pylori infection, tumour differentiation, disease stage or survival, most likely because of the low numbers involved. Thus, PEA3 protein expression is
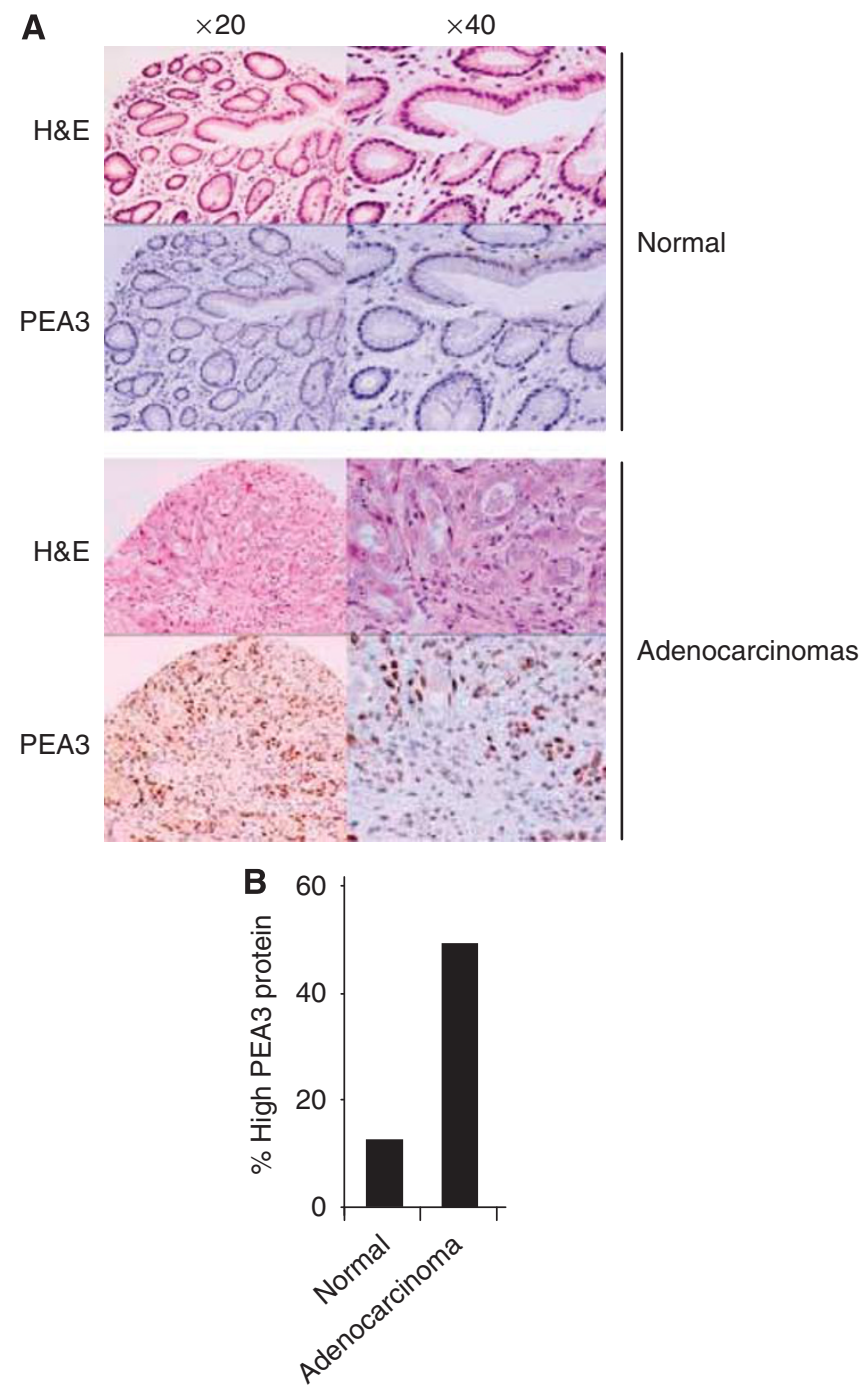

Figure I PEA3 protein expression in gastric adenocarcinomas. (A) Examples of typical TMA staining (brown stain) for PEA3 protein in normal gastric epithelium and adenocarcinoma (top panels H\&E, bottom panels PEA3 staining) at $\times 20$ and $\times 40$ magnification. (B) Summary of TMA data for moderate - high PEA3 protein expression (scored as intensity 3 or 4 ) in patient samples from normal and adenocarcinoma tissue classes. associated with gastric adenocarcinomas in a Western population, which is consistent with similar findings in mRNA expression levels (Wu et al, 2006) and protein levels in Japanese populations (Yamamoto et al, 2004).

\section{PEA3 and ER81 are associated with $M M P-1$ and $M M P-7$ expression}

To further extend the immunohistochemistry results, the levels of PEA3 and ER81 mRNA were analysed in a subset of the normal and adenocarcinoma-derived samples from patients. Samples from normal tissue exhibited low-level expression of these two transcription factors but a large number of tumour-derived samples exhibited elevated expression of PEA3 and/or ER81 (Figures 2A and B; summarised in Figure 2E). The overall median and the distribution of PEA3 and ER81 expression was 11- and 12-fold higher, respectively, in gastric cancer tissue compared with non-cancer tissue ( $P$-values 0.080 and 0.045 , respectively) (Figures $2 \mathrm{~F}$ and $\mathrm{G})$. Elevated PEA3 and ER81 mRNA expression was seen in 42 and 79\%, respectively, of gastric adenocarcinomas, which was more often than observed in normal tissue. In the case of ER81, the elevated frequency in tumours reached statistical significance ( $P$-values 0.063 and 0.008 , respectively).

PEA3 has previously been shown to regulate $M M P-1$ and $M M P-7$ expression in gastric cancer cell lines (Yamamoto et al, 2004; Wu et al, 2006). In Japanese populations, PEA3 expression has been associated with $M M P-7$ expression in gastric adenocarcinomas (Yamamoto et al, 2004), and high average levels of PEA3 and $M M P-1$ expression have also been observed (Wu et al, 2006). We therefore tested whether that PEA3 might be involved in regulating $M M P-1$ and MMP-7 expression in our population. Samples from normal tissue contained very low levels of mRNAs encoding these two MMPs (Figures 2C and D; summarised in Figure 2E). In contrast, elevated levels of mRNAs encoding both MMPs were observed in samples from gastric adenocarcinomas, which were particularly marked for $M M P-1$, where increased expression was observed in $64 \%$ of gastric cancers (Figures 2C and D; summarised in Figure 2E). Importantly, in all cases, elevated levels of $M M P-1$ expression in adenocarcinomas were associated with elevated levels of PEA3 and/or ER81 (Figure 2E). A similar association was seen for elevated MMP-7 expression. These data are therefore consistent with a role for PEA3 and/or ER81 in regulating $M M P$ expression, although overexpression of the transcription factors alone appears insufficient for inducing $M M P$ expression, as there are cases where $P E A 3$ and/or ER81 are expressed but either $M M P-1$ and/or $M M P-7$ are not overexpressed (Figure 2E). Because of the small number of tumours that do not express elevated expression of $P E A 3$ and/or ER81, it is not possible to determine whether the lack of $M M P$ expression in these tumours is statistically significant. Similarly, we do not have any tumour samples that express MMP1 and/or MMP7 and lack PEA3 and/or ER81, which further exacerbates the inability to determine statistical significance of the observed associations. Nevertheless, our results show a strong association between the expression of PEA3 and/or ER81 and downstream MMPs.

Thus, PEA3 and ER81 are often upregulated at the mRNA level in gastric adenocarcinomas, which is consistent with the results of TMA analysis demonstrating overexpression of PEA3 protein in this cancer type. Further, correlations with $M M P$ expression suggest that PEA3 family members may play an important role in controlling these important mediators of tumour metastasis in this context.

\section{ERK MAPK signalling in combination with PEA3 expression is a marker of poor prognosis}

The influence of ERK MAP kinase signalling on gastric cancer is poorly understood. As ERK MAP kinase signalling is an important 

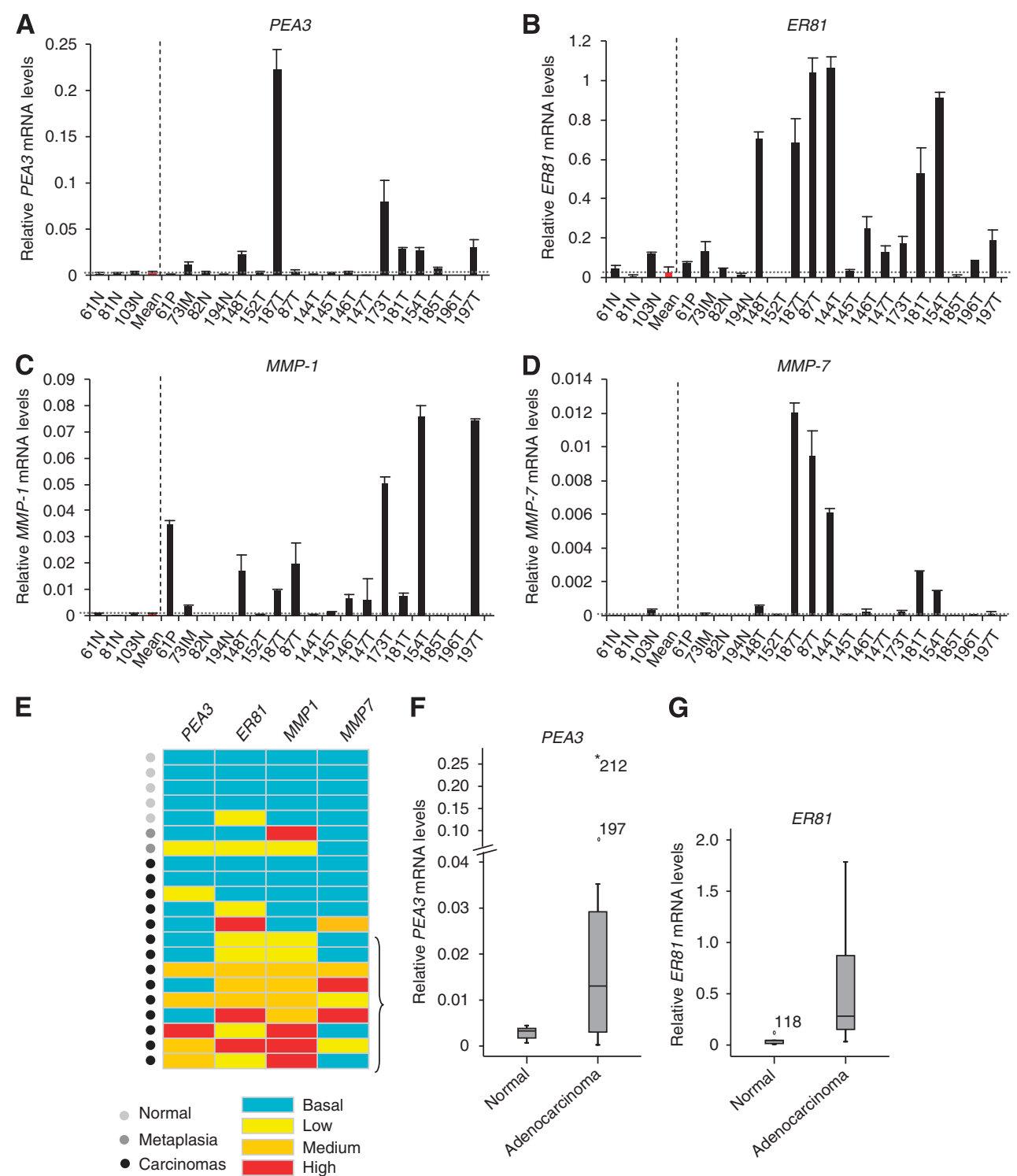

Figure 2 Correlative expression of PEA3 and ER8I with MMP-I and MMP-7 in gastric tissue specimens. (A-D) mRNA levels of PEA3 (A), ER8I (B), MMP-I (C) and MMP-7 (D) relative to I8S mRNA in tissue specimens are presented. All samples were standardised to mRNAs isolated from SW480 (for PEA3), Flol (for ER8I) or OE33 (for MMPs) cell lines. The average relative mRNA levels and standard deviations derived from at least two readings from one sample are shown. The individual tissue specimens are numbered. The samples are grouped according to the gastric tissue sub-types of normal gastric epithelium (N), adenomatous polyp (P), intestinal metaplasia (IM) and adenocarcinomas $(T)$. The average mRNA levels of the normal samples is shown in red and indicated by the horizontal dotted line. (E) Heat map summary of the relative mRNA levels of PEA3, ER8I, MMPI and MMP7 in the gastric tissue samples. Samples are categorised as 'normal' (light grey dots), 'metaplasia (dark grey dots) or from patients with oesophageal adenocarcinomas (black dots). Expression was defined as mRNA levels more than one standard deviation above the mean for the normal samples for each gene. Basal levels are anything below this value (turquoise rectangles). Expression was then divided into three categories relative to the mean; high expression was anything more than 100-fold (red rectangles), medium was between 25-100-fold (orange rectangles) and low was up to 25 fold (yellow rectangles) over the mean. Groups of samples showing increased expression of MMP-I and either PEA3 or ER8 I relative to basal levels are bracketed. (F and $\mathbf{G})$ Box plots of PEA3 (F) and ER8I (G) mRNA expression in oesophageal tissue taken from normal healthy controls and gastric adenocarcinoma patients. Median relative expression levels of PEA3 and ER8I are indicated for each tissue type. The box plot represents the inter-quartile range and the median value is indicated by the horizontal line. The $y$ axes in $(\mathbf{F})$ is split and the high outliers are labelled by case number.

upstream activator of members of the PEA3 subfamily, we therefore tested whether ERK signalling was activated in gastric adenocarcinoma samples and whether this signalling was associated with PEA3 subfamily member expression and subsequent disease progression. An antibody raised against the activated P-ERK was used to stain TMAs derived from gastric adenocarcinoma samples to test for active ERK signalling. Examples of the nuclear staining patterns of P-ERK in different classes of tissue are illustrated in Figure 3A. We tested the same 39 gastric cancer specimens and 15 non-cancer tissue specimens that we examined for PEA3 expression. Active ERK signalling was observed in both non-cancer tissue and cancer tissue, 79\% compared with 54\% (Figure 3B) reflecting ERK signalling as a normal physiological event in this tissue. However, subgroup analysis performed on the 39 gastric cancer specimens demonstrated that there were clear differences in the levels of ERK signalling among cancer samples (Figures $3 \mathrm{~A}$ and $\mathrm{C}$ ). Specimens with active ERK MAP kinase signalling were more frequently seen in tumours with metastases 

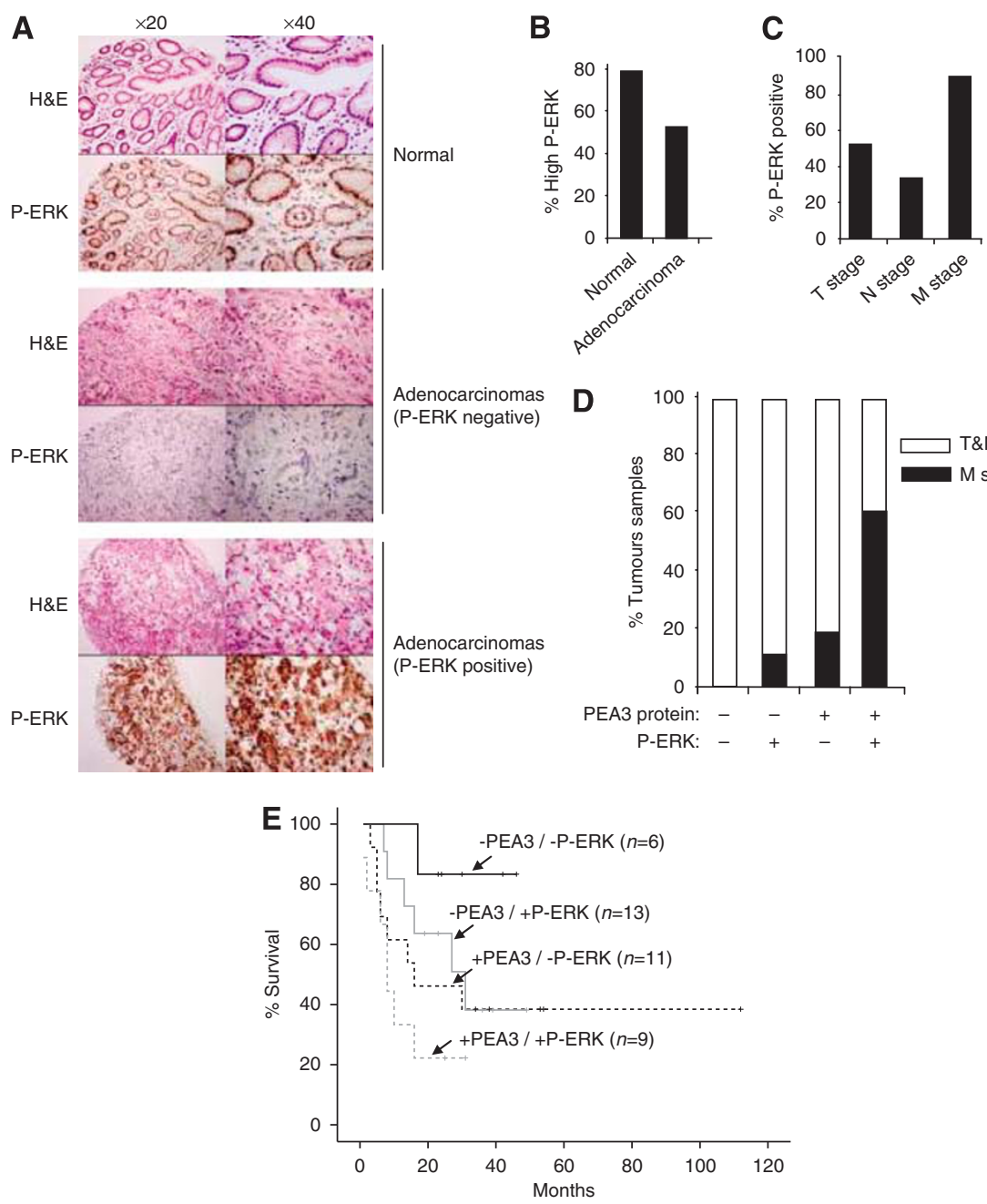

Figure 3 Correlative expression of PEA3 with high levels of ERK MAPK signalling in gastric adenocarcinoma specimens. (A) Examples of typical TMA staining (brown stain) for phosphorylated active ERK (P-ERK) levels in normal gastric epithelium, negative adenocarcinoma and positive adenocarcinoma (top panels H\&E, bottom panels P-ERK staining) at $\times 20$ and $\times 40$ magnification. (B) Summary of TMA data for moderate-high P-ERK signalling in patient samples from normal and adenocarcinoma tissue classes. Positive ERK MAP kinase signalling is defined as more than $5 \%$ tumour cells staining positive for P-ERK at intensity 3-4 at $\times 20$ magnification. (C) Histograms correlating the percentage of patients with TNM stage and phospho-ERK (P-ERK) levels (as defined in B). (D) Patients with a combination of higher than basal P-ERK levels and above basal PEA3 protein expression are correlated with metastatic M-stage disease. Positive P-ERK signals and PEA3 protein presence are defined as in (B). Data are presented as the percentage of patients containing the indicated combinations of P-ERK and PEA3 levels, which have T and N (white bars) or M-stage (black bars) tumours. (E) Patients with the indicated combinations of basal level $(-)$, and above basal P-ERK levels and PEA3 protein expression $(+)$ are correlated with \% survival. The numbers of cases with each combination of markers are shown in brackets.

(Figure $3 \mathrm{C} ; P$-value $=0.040$ ) and median survival was significantly worse, 10 months compared with 27 months in samples with lower ERK MAP kinase signalling activity $(P$-value $=0.050)($ data not shown).

Next, we investigated if active ERK MAP kinase signalling influenced the clinical outcomes in relation to PEA3 expression. In cultured gastric cancer cells, it has been shown that ERK MAP kinase signalling enhances PEA3 and MMP-1 expression (Wu et al, 2006). In gastric adenocarcinoma tissue, we have identified an association between PEA3/ER81 and MMP-1 expression. We therefore postulated that ERK signalling might influence clinical features of gastric adenocarcinoma patients in relation to PEA3 expression either directly via influencing $M M P$ expression or through some other mechanism. The expression of elevated levels of PEA3 protein and P-ERK signal was therefore compared among the 39 tumour samples (Figure 3D). Tumours with little PEA3 protein expression and low P-ERK levels were all of the T\&N stage class. Conversely, tumours with a dual combination of elevated PEA3 protein and P-ERK levels were more likely to have distant metastases ( $M$ stage) than be at the $T \& N$ stage. Indeed, this association with $M$ stage was much stronger with this dual combination compared with tumours with low expression or activation of one factor in isolation (Figure $3 \mathrm{D} ; \chi^{2} P$-value $=0.038$ ). Furthermore, a similar pattern is observed in patient survival where the Kapplan-Meier graph shows a clear relationship between PEA3 levels, P-ERK levels and patient survival (Figure 3E). Patient survival is worse in the presence of elevated levels of either PEA3 protein or P-ERK and even worse when both levels are elevated together. However, in comparison with the double-negative population neither P-ERK alone $(P$-value $=0.072)$ or PEA3 expression alone $(P$-value $=0.199)$ reached statistical significance compared with the significant association seen with patients double positive for P-ERK and PEA3 expression $(P$-value $=0.019)$. Conversely, in comparison with the double-positive population neither alone $(P$-value $=0.345)$ nor PEA3 expression alone $(P$-value $=0.053)$ reached statistical significance. Thus, only the presence of elevated levels of both 
PEA3 and P-ERK provides a reliable indicator of worse patient prognosis.

Together these results indicate that although the occurrence of elevated P-ERK in tumours is associated with late-stage disease, the combination of elevated P-ERK levels and PEA3 expression is more strongly associated with advanced-stage metastatic disease and shorter survival.

\section{DISCUSSION}

Members of the PEA3 subfamily are associated with a variety of cancers including colon, breast, ovarian, prostate and oesophageal cancer (de Launoit et al, 2000; Horiuchi et al, 2003; Cowden Dahl et al, 2007; Tomlins et al, 2007; Chi et al, 2010; Keld et al, 2010). Here, we have demonstrated that increased expression of PEA3 and ER81 is associated with gastric adenocarcinoma in a Western population and this is consistent with previous studies on an Asian population (Yamamoto et al, 2004). Moreover, we have shown a relationship between PEA3 and ER81 expression with the expression of $M M P-1$ and, to a lesser extent, $M M P-7$ in gastric adenocarcinomas. This mirrors the findings in gastric cancer cell lines and in gastric cancer biopsies derived from Japanese populations where PEA3 and MMP-1 overexpression was also observed (Wu et al, 2006) or where an association between PEA3 and $M M P-7$ expression was thought to be more important for cancer cell invasion (Yamamoto et al, 2004). In addition, we have presented data for PEA3 expression in all stages of gastric adenocarcinoma in our population whereas these previous findings were mainly focussed on early gastric cancer. Our data suggest that both PEA3 and ER81 are related to MMP-1 and MMP-7 expression. Although the RNA analysis is underpowered and larger studies are needed, the findings indicate that an inter-play between PEA3 and ER81 may exist in relation to expression of $M M P-1$. Other ETS proteins including ETS1, GABP $\alpha$ and Elk-1 are known to bind and regulate the same gene promoters (Hollenhorst et al, 2007; Boros et al, 2009). It is plausible that this mechanism may also be operative with PEA3 and ER81 in different gastric adenocarcinomas.

Importantly, our results indicate that PEA3 mRNA or protein expression in isolation is insufficient to specify cancer progression, as no relationship was demonstrated with disease stage or prognosis. Mechanisms derived from cell lines inform us that PEA3 needs activation for optimal promoter transactivation activity. A model of PEA3 activation by $H$. Pylori through ERK MAP kinase signalling has been suggested in gastric cancer cells and a similar mechanism of ERK activation appears to be necessary for PEA3 activity in oesophageal and ovarian cancer cell lines (Wu et al, 2006; Cowden Dahl et al, 2007; Keld et al, 2010). More recently, studies in gastrointestinal stromal tumours indicate that c-KIT-mediated upregulation of ERK signalling is a pre-requisite for the stabilisation of ER81 at the protein level and the subsequent promotion of tumourigenesis (Chi et al, 2010). Our studies suggest that similar mechanisms might also operate in gastric adenocarcinomas as tumours with combinations of high PEA3 and P-ERK levels fare worse than those with low levels of one or both of these molecular events. However, we do not see an obvious correlation between P-ERK and levels of PEA3 protein expression in cancer samples, but this would likely only be revealed by more quantitative western analysis rather than the more subjective TMA analysis. Our data suggest that an axis of ERK-PEA3/ER81-MMP-1/7 exists in these tumours as observed in oesophageal adenocarcinomas (Keld et al, 2010) but this is not confirmed in the current study, which is limited by design and patient number. However, the information from the current study may be important in tailoring cancer therapy in the future. We have shown that PEA3 upregulation in isolation does not predict prognosis in any stage of gastric cancer but that simultaneous upregulation of PEA3 expression and ERK pathway signalling is indicative of late-stage disease. Our data suggest a role for targeting cancer therapies to tumours not only with an active signalling pathway but also ones with a highly expressed transcription factors known to regulate key carcinogenic genes. Indeed, there are clearly two distinct gastric cancer populations with high and low levels of active ERK, but importantly, normal gastric tissue samples have high levels of active ERK, implying that downregulation of ERK signalling occurs during tumourigenesis with subsequent re-activation at some point, which associates with late-stage disease. Clinical drug trials that inhibit the ERK pathway at the growth factor receptor level (e.g., EGFR) or at the signal transducer protein level (e.g., MEK) have yielded limited results (Rinehart et al, 2004; Rojo et al, 2006). The identification of additional signal-dependant transcription factors that influence prognosis may improve future outcomes.

\section{ACKNOWLEDGEMENTS}

We thank Karren Palmer for excellent technical assistance; members of our laboratory for stimulating discussions; Ian Donaldson for help with data visualisation. We thank Catherine West and Helen Valentine for their assistance with RNA tissue specimens, Susan Pritchard (UHSM FT, Manchester, UK), David Harrison, Craig Rodgers (WWL FT, Wigan, UK) and Stephen Hayes (SRH FT, Salford, UK) for their help with tissue dissection and location of archived paraffin tissue specimens, Ian Welch, Simon Galloway (UHSM FT, Manchester, UK), Laura Formela and John Vickers (SRH FT, Salford, UK) for their assistance in retrieving surgical specimens. This work was supported by grants from Research and Development (ABJ0026) and the Cancer Therapy and Research Fund from the WWL NHS foundation trust and from the department of Gastroenterology to RK and YSA, the UK NIHR/UKCRN (UK National Institute of Health Research/ Cancer Research Network) to YSA and grants from the Wellcome Trust and a Royal Society-Wolfson award to ADS.

\section{REFERENCES}

Bao W, Fu HJ, Jia LT, Zhang Y, Li W, Jin BQ, Yao LB, Chen SY, Yang AG (2010) HER2-mediated upregulation of MMP-1 is involved in gastric cancer cell invasion. Arch Biochem Biophys 499: 49-55

Boros J, O’Donnell A, Donaldson IJ, Kasza A, Zeef L, Sharrocks AD (2009) Overlapping promoter targeting by Elk-1 and other divergent ETSdomain transcription factor family members. Nucleic Acids Res 37: $7368-7380$

Bosetti C, Bertuccio P, Levi F, Lucchini F, Negri E, La Vecchia C (2008) Cancer mortality in the European Union, 1970-2003, with a joinpoint analysis. Ann Oncol 19: 631-640

Brown LA, Amores A, Schilling TF, Jowett T, Baert JL, de Launoit Y, Sharrocks AD (1998) Molecular characterization of the zebrafish PEA3 ETS-domain transcription factor. Oncogene 17: 93-104 
factors synergizes with beta-catenin-LEF-1 to activate matrilysin transcription in intestinal tumors. Mol Cell Biol 21: 1370-1383

Cunningham D, Allum WH, Stenning SP, Thompson JN, Van de Velde CJ, Nicolson M, Scarffe JH, Lofts FJ, Falk SJ, Iveson TJ, Smith DB, Langley RE, Verma M, Weeden S, Chua YJ, Participants MT (2006) Perioperative chemotherapy $v s$ surgery alone for resectable gastroesophageal cancer. $N$ Engl J Med 355: 11-20

de Launoit Y, Baert JL, Chotteau-Lelievre A, Monte D, Coutte L, Mauen S, Firlej V, Degerny C, Verreman K (2006) The Ets transcription factors of the PEA3 group: transcriptional regulators in metastasis. Biochim Biophys Acta 1766: 79-87

de Launoit Y, Baert JL, Chotteau A, Monte D, Defossez PA, Coutte L, Pelczar H, Leenders F (1997) Structure-function relationships of the PEA3 group of Ets-related transcription factors. Biochem Mol Med 61: $127-135$

de Launoit Y, Chotteau-Lelievre A, Beaudoin C, Coutte L, Netzer S, Brenner C, Huvent I, Baert JL (2000) The PEA3 group of ETS-related transcription factors. Role in breast cancer metastasis. Adv Exp Med Biol 480: 107-116

Feng W, Brown RE, Trung CD, Li W, Wang L, Khoury T, Alrawi S, Yao J, Xia K, Tan D (2008) Morphoproteomic profile of mTOR, Ras/Raf kinase/ ERK, and NF-kappaB pathways in human gastric adenocarcinoma. Ann Clin Lab Sci 38: $195-209$

Findlay M, Cunningham D, Norman A, Mansi J, Nicolson M, Hickish T, Nicolson V, Nash A, Sacks N, Ford H, Carter R, Hill A (1994) A phase II study in advanced gastro-esophageal cancer using epirubicin and cisplatin in combination with continuous infusion 5-fluorouracil (ECF). Ann Oncol 5: 609-616

Goel A, Janknecht R (2004) Concerted activation of ETS protein ER81 by p160 coactivators, the acetyltransferase p300 and the receptor tyrosine kinase HER2/Neu. J Biol Chem 279: 14909-14916

Gum R, Lengyel E, Juarez J, Chen JH, Sato H, Seiki M, Boyd D (1996) Stimulation of $92-\mathrm{kDa}$ gelatinase B promoter activity by ras is mitogenactivated protein kinase kinase 1-independent and requires multiple transcription factor binding sites including closely spaced PEA3/ets and AP-1 sequences. J Biol Chem 271: $10672-10680$

Guo B, Sallis RE, Greenall A, Petit MM, Jansen E, Young L, Van de Ven WJ, Sharrocks AD (2006) The LIM domain protein LPP is a coactivator for the ETS domain transcription factor PEA3. Mol Cell Biol 26: 4529-4538

Guo B, Sharrocks AD (2009) Extracellular signal-regulated kinase mitogenactivated protein kinase signaling initiates a dynamic interplay between sumoylation and ubiquitination to regulate the activity of the transcriptional activator PEA3. Mol Cell Biol 29: 3204-3218

Guo B, Yang SH, Witty J, Sharrocks AD (2007) Signalling pathways and the regulation of SUMO modification. Biochem Soc Trans 035: 1414-1418

Habelhah H, Okada F, Kobayashi M, Nakai K, Choi S, Hamada J, Moriuchi T, Kaya M, Yoshida K, Fujinaga K, Hosokawa M (1999) Increased E1AF expression in mouse fibrosarcoma promotes metastasis through induction of MT1-MMP expression. Oncogene 18: 1771-1776

Hollenhorst PC, Shah AA, Hopkins C, Graves BJ (2007) Genome-wide analyses reveal properties of redundant and specific promoter occupancy within the ETS gene family. Genes Dev 21: 1882-1894

Horiuchi S, Yamamoto H, Min Y, Adachi Y, Itoh F, Imai K (2003) Association of ets-related transcriptional factor E1AF expression with tumour progression and overexpression of MMP-1 and matrilysin in human colorectal cancer. J Pathol 200: 568-576

Howe LR, Crawford HC, Subbaramaiah K, Hassell JA, Dannenberg AJ, Brown AM (2001) PEA3 is up-regulated in response to Wnt1 and activates the expression of cyclooxygenase-2. J Biol Chem 276: 20108-20115

Hua D, Chen B, Bai M, Yu H, Wu X, Jin W (2009) PEA3 activates VEGF transcription in T47D and SKBR3 breast cancer cells. Acta Biochim Biophys Sin (Shanghai) 41: 63-68

Janknecht R (2003) Regulation of the ER81 transcription factor and its coactivators by mitogen- and stress-activated protein kinase 1 (MSK1). Oncogene 22: $746-755$

Jiang J, Wei Y, Liu D, Zhou J, Shen J, Chen X, Zhang S, Kong X, Gu J (2007) E1AF promotes breast cancer cell cycle progression via upregulation of Cyclin D3 transcription. Biochem Biophys Res Commun 358: 53-58

Keld R, Guo B, Downey P, Gulmann C, Ang YS, Sharrocks AD (2010) The ERK MAP kinase-PEA3/ETV4-MMP-1 axis is operative in oesophageal adenocarcinoma. Molecular Cancer 9: 313
Liu Y, Borchert GL, Phang JM (2004) Polyoma enhancer activator 3, an ets transcription factor, mediates the induction of cyclooxygenase- 2 by nitric oxide in colorectal cancer cells. J Biol Chem 279: 18694-18700

Mackay HJ, McInnes A, Paul J, Raby N, Lofts FJ, McDonald AC, Soukop M, Fullarton GM, Harris AL, Garcia-Vargas J, Evans TR (2001) A phase II study of epirubicin, cisplatin and raltitrexed combination chemotherapy (ECT) in patients with advanced oesophageal and gastric adenocarcinoma. Ann Oncol 12: $1407-1410$

Matsui K, Sugimori K, Motomura H, Ejiri N, Tsukada K, Kitajima I (2006) PEA3 cooperates with c-Jun in regulation of HER2/neu transcription. Oncol Rep 16: $153-158$

Nosho K, Yoshida M, Yamamoto H, Taniguchi H, Adachi Y, Mikami M, Hinoda Y, Imai K (2005) Association of Ets-related transcriptional factor E1AF expression with overexpression of matrix metalloproteinases, COX-2 and iNOS in the early stage of colorectal carcinogenesis. Carcinogenesis 26: $892-899$

O'Hagan RC, Hassell JA (1998) The PEA3 Ets transcription factor is a downstream target of the HER2/Neu receptor tyrosine kinase. Oncogene 16: $301-310$

Oikawa T, Yamada T (2003) Molecular biology of the Ets family of transcription factors. Gene 303: $11-34$

Rachet B, Maringe C, Nur U, Quaresma M, Shah A, Woods LM, Ellis L, Walters S, Forman D, Steward J, Coleman MP (2009) Population-based cancer survival trends in England and Wales up to 2007: an assessment of the NHS cancer plan for England. Lancet Oncol 10: 351-369

Rinehart J, Adjei AA, Lorusso PM, Waterhouse D, Hecht JR, Natale RB, Hamid O, Varterasian M, Asbury P, Kaldjian EP, Gulyas S, Mitchell DY, Herrera R, Sebolt-Leopold JS, Meyer MB (2004) Multicenter phase II study of the oral MEK inhibitor, CI-1040, in patients with advanced nonsmall-cell lung, breast, colon, and pancreatic cancer. J Clin Oncol 22: $4456-4462$

Rojo F, Tabernero J, Albanell J, Van Cutsem E, Ohtsu A, Doi T, Koizumi W, Shirao K, Takiuchi H, Ramon y Cajal S, Baselga J (2006) Pharmacodynamic studies of gefitinib in tumor biopsy specimens from patients with advanced gastric carcinoma. J Clin Oncol 24: 4309-4316

Samalin E, Ychou M (2007) Neoadjuvant treatment in upper gastrointestinal adenocarcinomas: new paradigms from old concepts? Curr Opin Oncol 19: $384-389$

Sharrocks AD (2001) The ETS-domain transcription factor family. Nat Rev Mol Cell Biol 2: $827-837$

Tan YK, Fielding JW (2006) Early diagnosis of early gastric cancer. Eur J Gastroenterol Hepatol 18: 821-829

Tebbutt NC, Norman A, Cunningham D, Iveson T, Seymour M, Hickish T, Harper P, Maisey N, Mochlinski K, Prior Y, Hill M (2002) A multicentre, randomised phase III trial comparing protracted venous infusion (PVI) 5-fluorouracil (5-FU) with PVI 5-FU plus mitomycin C in patients with inoperable oesophago-gastric cancer. Ann Oncol 13: $1568-1575$

Tomlins SA, Laxman B, Dhanasekaran SM, Helgeson BE, Cao X, Morris DS, Menon A, Jing X, Cao Q, Han B, Yu J, Wang L, Montie JE, Rubin MA, Pienta KJ, Roulston D, Shah RB, Varambally S, Mehra R, Chinnaiyan AM (2007) Distinct classes of chromosomal rearrangements create oncogenic ETS gene fusions in prostate cancer. Nature 448: 595-599

Vrieseling E, Arber S (2006) Target-induced transcriptional control of dendritic patterning and connectivity in motor neurons by the ETS gene Pea3. Cell 127: 1439-1452

Wu JY, Lu H, Sun Y, Graham DY, Cheung HS, Yamaoka Y (2006) Balance between polyoma enhancing activator 3 and activator protein 1 regulates Helicobacter pylori-stimulated matrix metalloproteinase 1 expression. Cancer Res 66: $5111-5120$

Yamamoto H, Horiuchi S, Adachi Y, Taniguchi H, Nosho K, Min Y, Imai K (2004) Expression of ets-related transcriptional factor E1AF is associated with tumor progression and over-expression of matrilysin in human gastric cancer. Carcinogenesis 25: 325-332

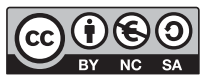

This work is licensed under the Creative Commons Attribution-NonCommercial-Share Alike 3.0 Unported License. To view a copy of this license, visit http://creativecommons. org/licenses/by-nc-sa/3.0/ 